\title{
Role of Dual-Energy Computed Tomography in Gallbladder Disease: A Review
}

\author{
Tarvinder Singh ${ }^{1}$ Pankaj Gupta ${ }^{1}$ \\ ${ }^{1}$ Department of Radiodiagnosis and Imaging, Postgraduate Institute \\ of Medical Education and Research (PGIMER), Chandigarh, India \\ J Gastrointestinal Abdominal Radiol ISGAR 2022;5:107-113.

\begin{abstract}
Address for correspondence Pankaj Gupta, MD, Department of Radiodiagnosis and Imaging, Postgraduate Institute of Medical Education and Research (PGIMER), Chandigarh 160012, India (e-mail: Pankajgupta959@gmail.com).
\end{abstract}

\begin{abstract}
Keywords

Gallbladder diseases are common and include a spectrum ranging from benign to cancer. Imaging plays an integral role in the diagnosis and in guiding appropriate management. While most patients with gallstone (GS) diseases can be evaluated with ultrasound, those with complicated GS disease, suspicion of cancer, or staging of cancer need additional cross-sectional imaging. Computed tomography (CT) is widely available and is often the imaging test of choice following an equivocal ultrasound or negative ultrasound in patients with unexplained symptoms. Conventional CT has limited sensitivity in detecting GS or common bile duct stones. In other scenarios, including diagnosis of acute cholecystitis (AC) and characterization of gallbladder wall thickening, an increase in accuracy using novel techniques is desirable. Dual-energy computed tomography (DECT) is increasingly incorporated

- gallbladder

- cancer

- dual-energy CT

- CT into clinical practice. DECT has shown promising results in the detection of cholesterol stones that otherwise go unnoticed on conventional CT. However, its role beyond GS disease has largely been unexplored. In this review, we discuss the available literature on the DECT in biliary diseases and discuss the potential applications of this technique.
\end{abstract}

\section{Introduction}

There is a spectrum of pathologies affecting the gallbladder (GB), ranging from benign to malignant. Among these conditions, gallstone (GS) disease is the commonest. ${ }^{1}$ Therefore, imaging plays an essential role in investigating patients suspected to have biliary diseases. Imaging modalities that are frequently utilized to evaluate biliary tract disease include ultrasonography (USG), computed tomography (CT), magnetic resonance cholangiopancreatography, and hepatobiliary scintigraphy. USG is a cost-effective and readily available technique for evaluating biliary diseases with esti- mated sensitivity and specificity for acute cholecystitis (AC) being 88 to $94 \%$ and 78 to $80 \%$, respectively. ${ }^{2} \mathrm{CT}$ also plays an essential role in diagnosing GB diseases especially complicated $\mathrm{AC}$ and suspected malignancy, although it is less sensitive than USG for the detection of $\mathrm{GS}^{3}{ }^{3}$ Dual-energy computed tomography (DECT) is a technical advancement that has been exploited for clinical applications in various organ systems and pathologies, including biliary diseases. In this article, we review the role of DECT in the evaluation of biliary disease.

DECT can overcome some of the limitations of conventional CT in the evaluation of GB diseases. DECT is based on published online February 18, 2022
DOI https://doi.org/

10.1055/s-0042-1743173. ISSN 2581-9933.
(C) 2022. Indian Society of Gastrointestinal and Abdominal Radiology. All rights reserved.

This is an open access article published by Thieme under the terms of the Creative Commons Attribution-NonDerivative-NonCommercial-License, permitting copying and reproduction so long as the original work is given appropriate credit. Contents may not be used for commercial purposes, or adapted, remixed, transformed or built upon. (https://creativecommons.org/ licenses/by-nc-nd/4.0/)

Thieme Medical and Scientific Publishers Pvt. Ltd., A-12, 2nd Floor, Sector 2, Noida-201301 UP, India 
the concept of scan acquisition with the help of different $\mathrm{X}$ ray energy levels: one acquired at higher $\mathrm{kVp}$ (usually $140 \mathrm{kVp}$ ) and the other at lower kVp (usually 80-100 kVp). We can extract the composition of a substance if we know how it interacts at different energy levels. The interaction that is most important for DECT application is the photoelectric effect. The increase in photoelectric absorption of X-ray photon, which is seen at energy levels higher than k-shell binding energy, is termed K-edge and forms the basis of dualenergy techniques. ${ }^{4}$ The most common methods for acquisition of DECT include dual source, single-source rapid kilovoltage switching, and dual-layer DECT (sandwich). Postprocessing techniques can be used to differentiate various structures based on their different X-ray absorption behavior as a function of X-ray energy. ${ }^{5}$ Virtual noncontrast (VNC) images can be generated when the iodine content is subtracted. Quantitative assessments, including the amount of iodine uptake and degree of enhancement, can be achieved from single CT image acquisition with the help of pure iodine map or overlay maps, where iodine content is superimposed. Atomic number map (Rho/Z) is a newly developed application of DECT that helps calculate the atomic number values in the lesion, thus allowing the separation of various materials.

\section{Role of DECT in Various Disease}

\section{Cholelithiasis}

GS disease affects 10 to $15 \%$ of the adult population, thus constituting a significant health problem. ${ }^{1}$ Individuals may remain asymptomatic throughout their life. The most common presentation is biliary colic that occurs due to obstruction by calculus at the cystic duct. Complications of GS include AC (ranging in severity from mild to severe, uncomplicated, or complicated), GS ileus, acute pancreatitis, and Mirizzi syndrome. Association of GS with GB cancer has also been reported in a few studies. Although USG is accurate in the diagnosis of GS, a CT scan is frequently utilized for routine workup. As the majority of GS are of cholesterol type (70\%), these may not be visible on CT as they are isoattenuating to surrounding bile. Dual-energy postprocessing techniques such as virtual monochromatic imaging (VMI) and VNC have shown positive results in improved detection of GS on DECT. ${ }^{6-11} \mathrm{Kim}$ et al found that VNC images were of equivalent quality as true unenhanced images. ${ }^{7} \mathrm{VNC}$ images showed increased contrast to noise ratio for the cholesterol GS compared with true unenhanced images. However, the visibility of calcified GS and smaller GS $(<9 \mathrm{~mm})$ was negatively affected due to the inaccurate decomposition of calcium and iodine. Thus, calcified GS are better visualized on true unenhanced images. ${ }^{7}$ In a study by Uyeda et al, VMI was useful in distinguishing GS from surrounding bile. ${ }^{8}$ The GS, which are of similar attenuation to bile on conventional CT (120 kVp), becomes more discernible on higher (200 keV) and lower (40 keV) keV VMI. The reason for better visualization is that GS has an energy-dependent X-ray attenuation curve different from surrounding bile. ${ }^{9}$ Noncalcified GS has lower attenuation than bile on low keV images and higher attenuation on high $\mathrm{keV}$ images. Low keV images have been found better in the detection of GS as the contrast difference among noncalcified GS, and surrounding bile is maximum compared with that at high keV images. Recently, Soesbe et al found that dual-layer DECT generation of the two-dimensional histogram of Compton and photoelectric attenuation was even more helpful in differentiating small $(<9 \mathrm{~mm})$ isoattenuating GS from the bile. ${ }^{10}$ Most materials have a different position in the two-dimensional histogram based upon their Compton and photoelectric ordered pairs. Thus, we can differentiate isoattenuating GS from bile (-Fig. 1). Atomic number map (Rho/Z) is another important application of DECT, which can help identify GS from surrounding fluid in the small bowel in cases of GS ileus. ${ }^{11}$

\section{Acute Cholecystitis}

In most cases, AC is caused by GS. Few cases occur secondary to stasis. ${ }^{12}$ USG is the initial imaging of choice for diagnosing

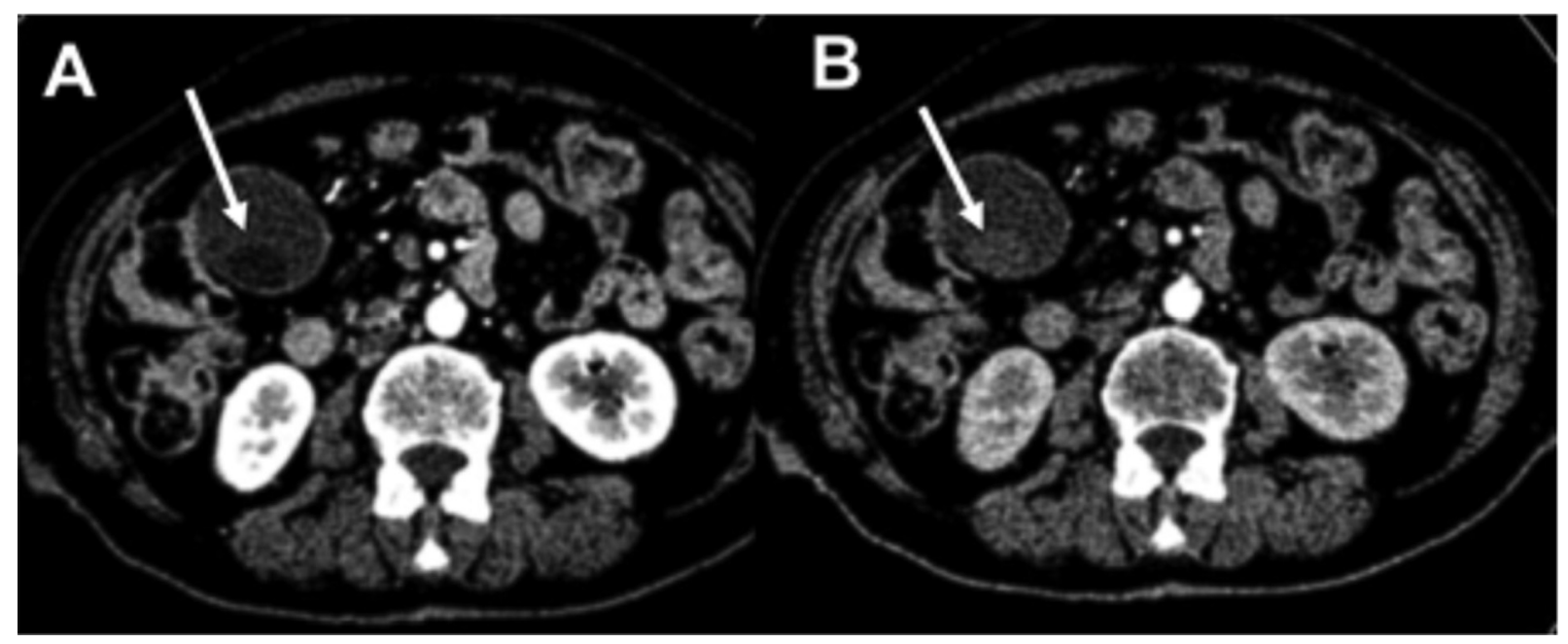

Fig. 1 Dual-energy computed tomography (DECT) in gallstone disease: (A, B) 80 keV, 140 keV DECT images show hypoattenuating calculus in gall bladder on $80 \mathrm{keV}$ images (arrow) and hyperattenuating calculus on $140 \mathrm{keV}$ images (arrow). 


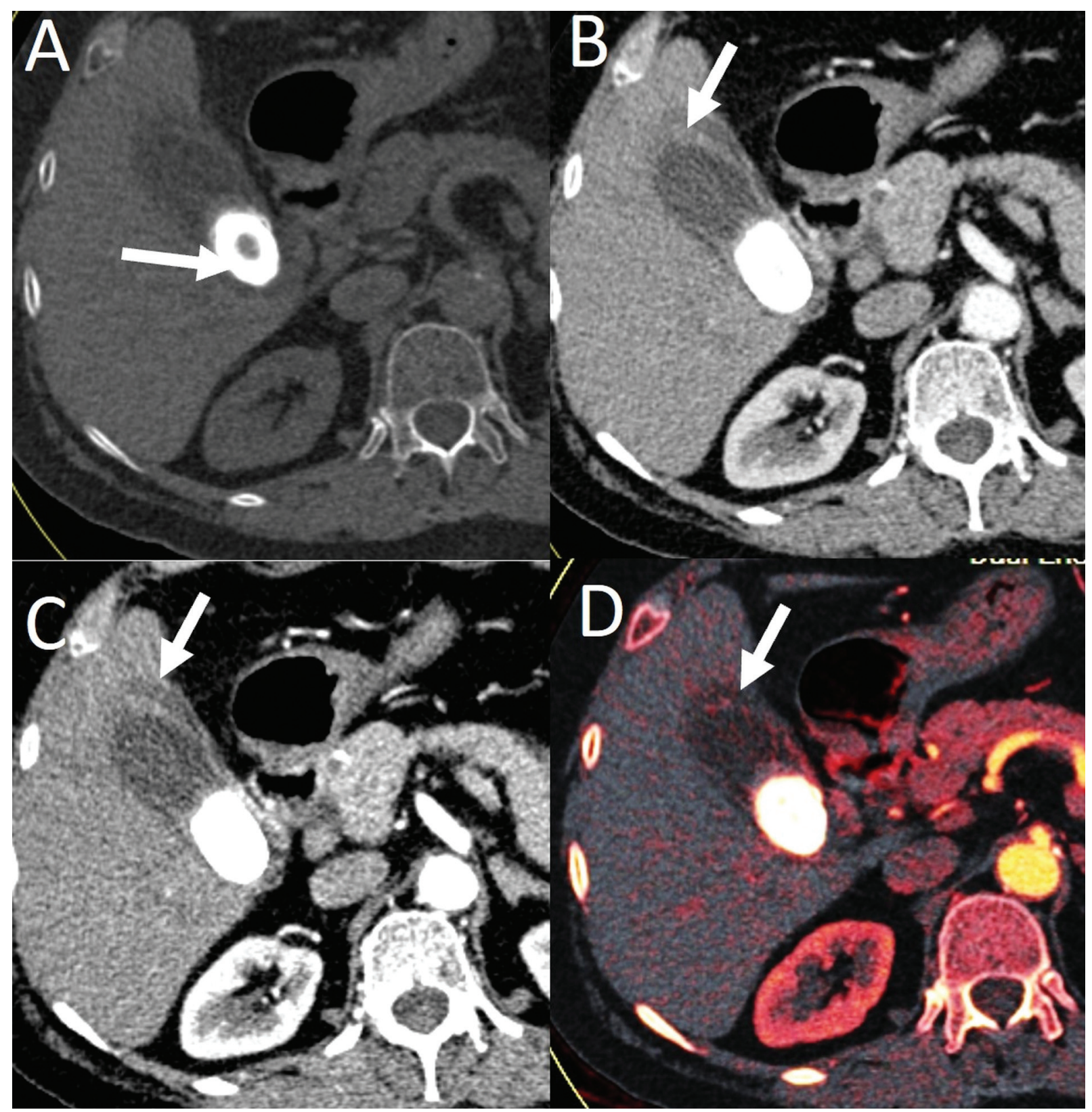

Fig. 2 Dual-energy computed tomography (CT) in acute cholecystitis. (A) Virtual nonenhanced CT image shows a large calculus impacted at the gallbladder neck (arrow). (B, C) $80 \mathrm{keV}$ and $140 \mathrm{keV}$ images show mural thickening and continuous mural enhancement (arrow). (D) lodine overlap image depicts the discontinuity of mural enhancement better (arrow). This may be a predictor of gallbladder perforation.

AC because of its high sensitivity. ${ }^{13}$ Patients whose diagnosis remains unclear on USG due to limitations of scanning or impacted stone in the neck or patients with complicated AC may undergo CT. ${ }^{14}$ DECT has the potential to be the single best imaging test for patients presenting with biliary colic because of its ability to identify GS and better visualization of findings of AC. ${ }^{15}$ Iodine maps generated from DECT can easily detect mural hyperemia and hypervascularity of the adjacent liver parenchyma (hot rim sign). Also, complications such as GB perforation may be better visualized using VMI and color-coded iodine maps where a nonenhancing defect in the GB wall can be easily detected (-Fig. 2).

\section{GB Polyp}

GB polyps are of many types, some of them have malignant potential, and others are generally benign, thus can be grouped into neoplastic (adenomatous) and nonneoplastic (cholesterol, inflammatory). USG has a high sensitivity for detecting polyps but cannot differentiate the histological type of GB polyps. Risk factors that help predict malignancy are size, female sex, elderly patient, number, and shape of the lesion. ${ }^{16}$ Based on previous studies, size $>1 \mathrm{~cm}$ of the lesion is the most important criteria for the likelihood of malignant polyp, but a lesion of size $<1 \mathrm{~cm}$ may also turn out to be adenomatous. ${ }^{17}$ Therefore, size cannot be reliably used as the only criteria to differentiate benign from malignant polyps. 


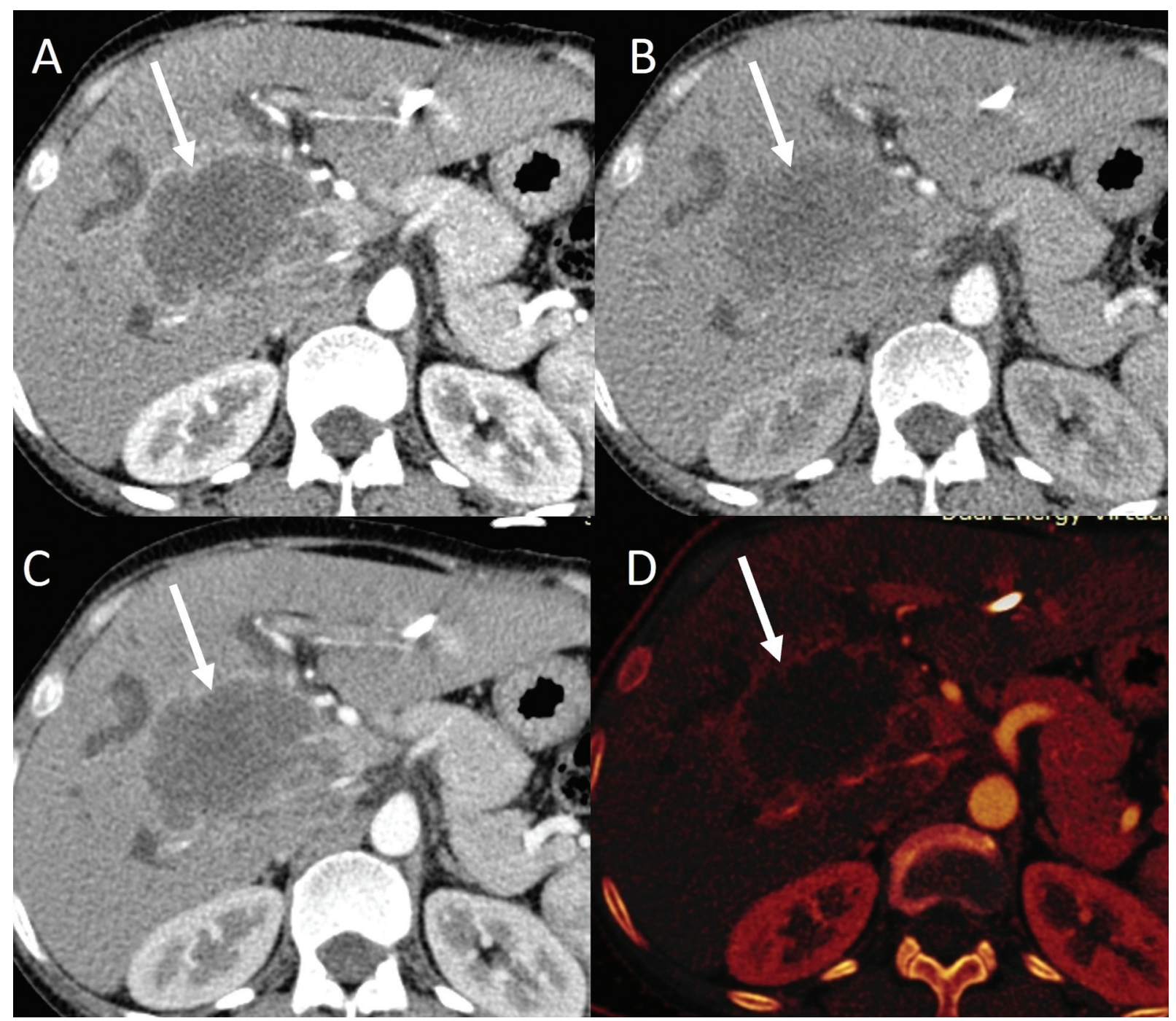

Fig. 3 Dual-energy computed tomography (DECT) in gallbladder cancer: mass replacing gallbladder. (A-C) $80 \mathrm{keV}, 140 \mathrm{keV}$, and mixed (representative of conventional CT) DECT images show a large mass replacing the gallbladder. The mass is necrotic and shows peripheral enhancement (arrow). (D) lodine overlay image (D) shows the peripheral iodine uptake (arrow).

Recently, few studies have shown DECT to be a promising modality that helps in diagnosis and differentiates the polyp types. In a study by Yin et al., different energy spectral curves were identified for cholesterol and adenomatous polyp. ${ }^{17}$ Cholesterol polyps showed positive mean attenuation value change, whereas adenomatous polyps had negative mean attenuation value change between $80-140 \mathrm{keV}$ or $40-$ $140 \mathrm{keV}$ VMI.

\section{GB Cancer}

GB cancer is the most common malignancy of the biliary system with a predilection for specific geographic regions of the world. ${ }^{18}$ The presentation is nonspecific. Most patients are diagnosed at an advanced stage with metastasis and have a poor prognosis. ${ }^{19}$ For the diagnosis and staging of GB cancer, imaging plays a crucial role. USG is the initial investigation of choice as it is cost-effective and readily available. However, CT and magnetic resonance imaging remain the preferred modalities for accurate diagnosis and staging. Morphologically, GB cancer has three main patterns; the most typical pattern is mass occupying or completely replacing the GB lumen (40-65\%), followed by focal or diffuse asymmetric GB wall thickening (20-30\%) and polypoid lesion (15-20\%) $)^{20}$ (- Fig. 3). Additional imaging findings such as associated lymphadenopathy, invasion of surrounding structures, and distant metastasis help in the diagnosis of wall thickening type of GB cancer.

\section{Benign Gallbladder Wall Thickening}

Benign conditions like adenomyomatosis, xanthogranulomatous cholecystitis, and cholecystitis may have similar findings on conventional CT. It is often difficult to accurately diagnose GB wall thickening. ${ }^{21-23}$ Hence, we require advanced imaging modalities to characterize GB wall thickening. DECT is a potential tool for this problem that has not been investigated. It may show the mural characteristics, including enhancement pattern and intramural cyst/hypodense nodules, better than conventional CT, 


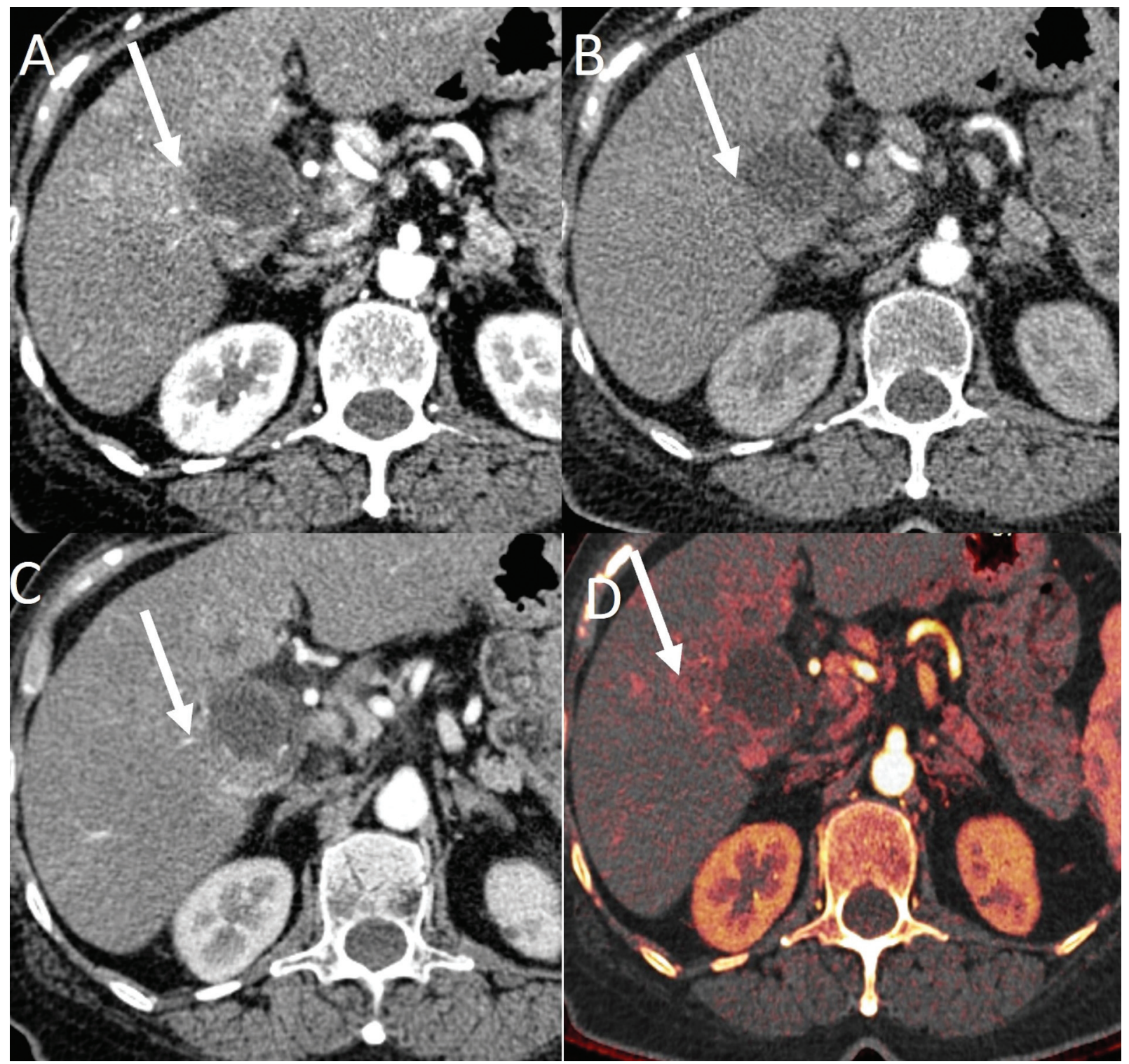

Fig. 4 Dual-energy computed tomography (DECT) in gallbladder cancer: wall thickening type. (A-C) $80 \mathrm{keV}, 140 \mathrm{keV}$, and mixed (representative of conventional CT) DECT images show mural thickening of the gallbladder body (arrow). Note that the enhancement characteristics are better seen at $80 \mathrm{keV}$ images. (D) lodine overlap image shows that the enhancement is asymmetric and heterogeneous (arrow) features favoring malignant nature.

especially on iodine maps. The presence of intramural cysts/Rokitansky-Aschoff sinuses is diagnostic for adenomyomatosis. The intactness of mucosa is critical for the diagnosis of benign GB wall thickening and is better assessed on iodine overlay maps. Finally, adjacent liver infiltration will be better seen on the iodine overlay images (-Fig. 4).

The critical characteristics of DECT studies evaluating biliary diseases are summarized in $\boldsymbol{- T a b l e ~} \mathbf{1}$.

\section{Future Directions}

DECT may provide quantitative data for texture analysis and radiogenomics in GB cancer. A recent study showed the incremental value of the radiomics model for detecting serosal invasion in preoperative restaging for locally advanced gastric cancer. ${ }^{24}$ Another study reported a DECTbased nomogram for detecting HER 2 status in gastric cancer. ${ }^{25}$ Furthermore, DECT may allow accurate differentiation of metastases from cholangitic abscess in the setting of GB cancer. This has enormous prognostic and therapeutic implications as metastatic GB cancer has a dismal prognosis and receives the best supportive care. In a recent study comprising 28 patients with multiple malignancies (GB cancer) and 23 patients with liver abscesses, DECT had sensitivity and specificity of 89.3 and $93.3 \%$ and area under the curve of 0.963 for differentiating liver abscess from metastases. ${ }^{26}$ The comparative performance of different DECT scanners/techniques needs full exploration before confident clinical applications. 


\begin{tabular}{|c|c|c|c|c|c|c|}
\hline 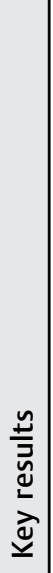 & 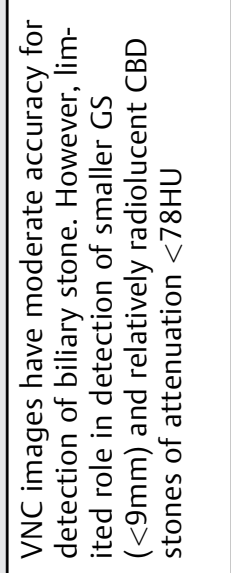 & 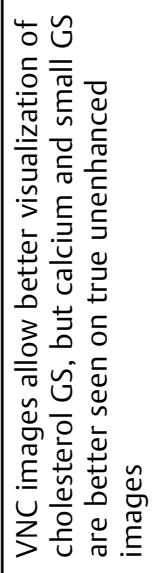 & 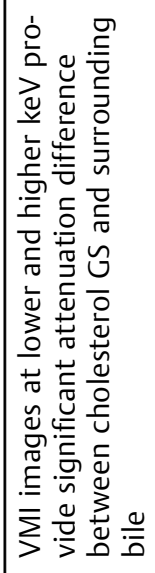 & 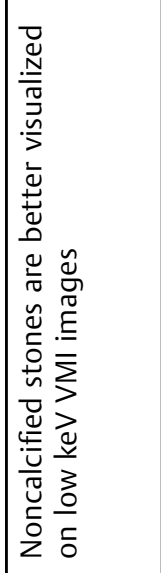 & 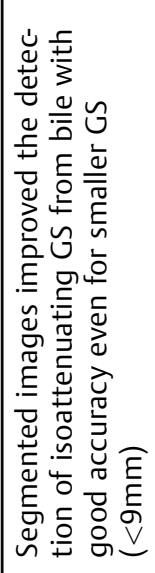 & 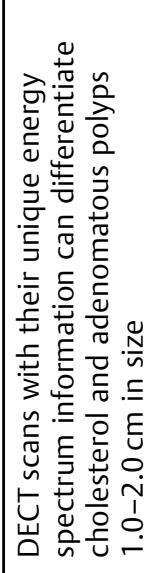 \\
\hline$\frac{E}{<}$ & 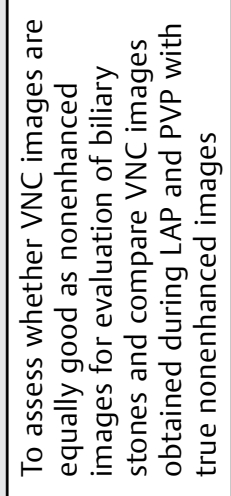 & 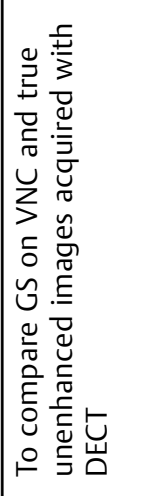 & 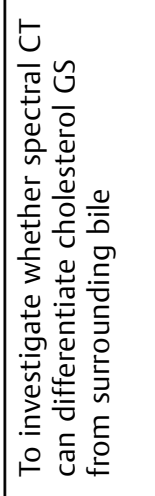 & 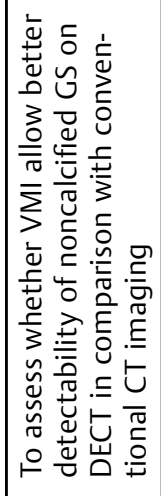 & 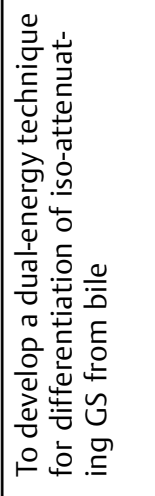 & 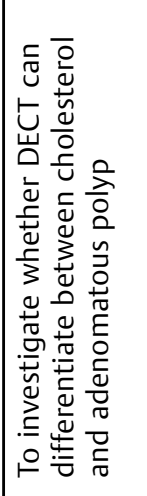 \\
\hline 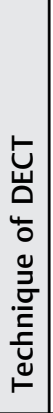 & 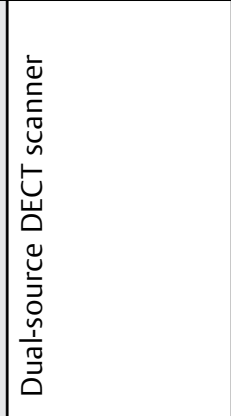 & 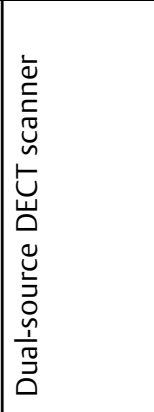 & 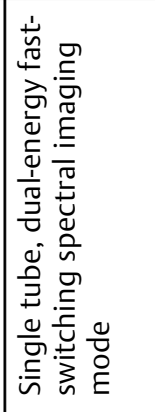 & 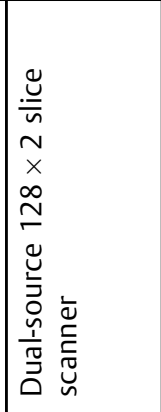 & 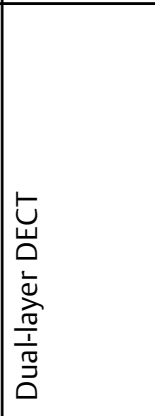 & 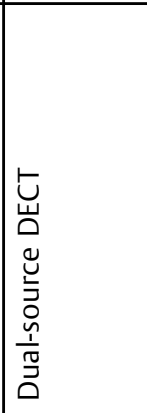 \\
\hline 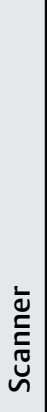 & 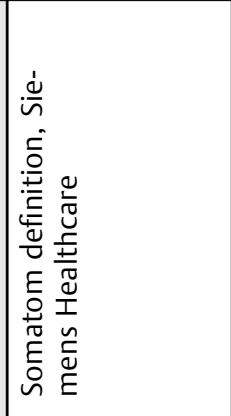 & 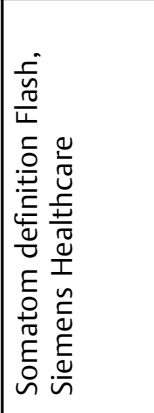 & 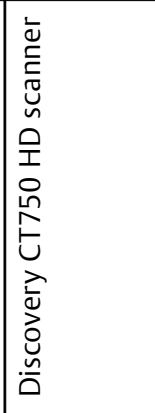 & 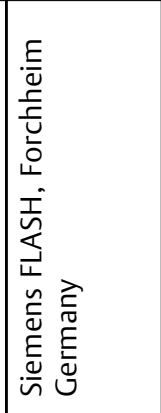 & 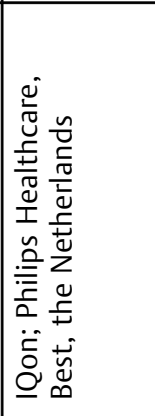 & 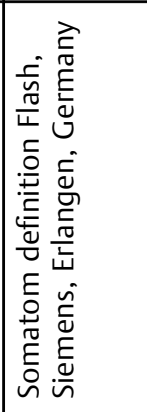 \\
\hline 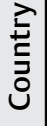 & 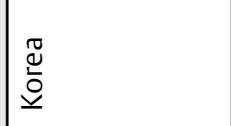 & \begin{tabular}{|l} 
J \\
$\underline{0}$ \\
$\underline{0}$
\end{tabular} & 胥 & 芩 & 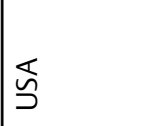 & 妾 \\
\hline 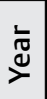 & $\overline{\bar{\nu}}$ & $\frac{\operatorname{mn}}{\stackrel{D}{\sim}}$ & $\frac{0}{\sim}$ & $\stackrel{\hat{\nu}}{\stackrel{\nu}{N}}$ & $\stackrel{a}{\grave{D}}$ & 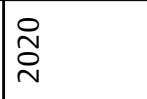 \\
\hline 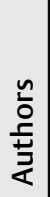 & $\mid \begin{array}{l}\bar{\sigma} \\
\tilde{U} \\
\tilde{U} \\
\underline{\underline{\underline{v}}}\end{array}$ & $\begin{array}{l}\frac{0}{\pi} \\
\pm \\
\pm \\
\Xi \\
\Xi\end{array}$ & 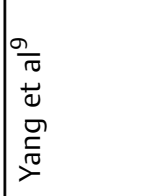 & 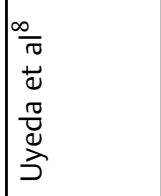 & 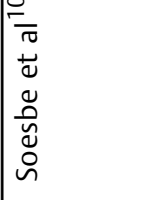 & 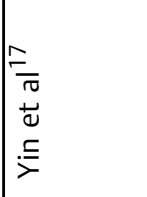 \\
\hline
\end{tabular}




\section{Conclusion}

DECT is a promising tool for the evaluation of biliary diseases. It has the potential to provide a one-stop solution to the detection of intraluminal and intramural pathologies, including differentiation of benign and malignant pathologies. However, there is limited data available on individual biliary tract pathologies. Therefore, future research must focus on knowing the potential of this application of CT scan.

Funding

None.

\section{Conflict of Interest}

None declared.

\section{References}

1 Russo MW, Wei JT, Thiny MT, et al. Digestive and liver diseases statistics, 2004. Gastroenterology 2004;126(05):1448-1453

2 Bennett GL, Balthazar EJ. Ultrasound and CT evaluation of emergent gallbladder pathology. Radiol Clin North Am 2003;41(06): 1203-1216

3 Barakos JA, Ralls PW, Lapin SA, et al. Cholelithiasis: evaluation with CT. Radiology 1987;162(02):415-418

4 Coursey CA, Nelson RC, Boll DT, et al. Dual-energy multidetector CT: how does it work, what can it tell us, and when can we use it in abdominopelvic imaging? Radiographics 2010;30(04): 1037-1055

5 Marin D, Boll DT, Mileto A, Nelson RC. State of the art: dual-energy CT of the abdomen. Radiology 2014;271(02):327-342

6 Lee HA, Lee YH, Yoon KH, Bang DH, Park DE. Comparison of virtual unenhanced images derived from dual-energy $\mathrm{CT}$ with true unenhanced images in evaluation of gallstone disease. AJR Am J Roentgenol 2016;206(01):74-80

7 Kim JE, Lee JM, Baek JH, Han JK, Choi BI. Initial assessment of dualenergy CT in patients with gallstones or bile duct stones: can virtual nonenhanced images replace true nonenhanced images? AJR Am J Roentgenol 2012;198(04):817-824

8 Uyeda JW, Richardson IJ, Sodickson AD. Making the invisible visible: improving conspicuity of noncalcified gallstones using dual-energy CT. Abdom Radiol (NY) 2017;42(12):2933-2939

9 Yang CB, Zhang S, Jia YJ, et al. Clinical application of dualenergy spectral computed tomography in detecting cholesterol gallstones from surrounding bile. Acad Radiol 2017;24(04): 478-482

10 Soesbe TC, Lewis MA, Xi Y, et al. A technique to identify isoattenuating gallstones with dual-layer spectral CT: an ex vivo phantom study. Radiology 2019;292(02):400-406
11 Liu P, Tan XZ. Dual-energy CT of gallstone ileus. Radiology 2020; 295(03):516-516

12 Gore RM, Thakrar KH, Newmark GM, Mehta UK, Berlin JW. Gallbladder imaging. Gastroenterol Clin North Am 2010;39(02):265-287 , ix ix.

13 Kiewiet JJS, Leeuwenburgh MMN, Bipat S, Bossuyt PMM, Stoker J, Boermeester MA. A systematic review and meta-analysis of diagnostic performance of imaging in acute cholecystitis. Radiology 2012;264(03):708-720

14 Young N, Kinsella S, Raio CC, et al. Economic impact of additional radiographic studies after registered diagnostic medical sonographer (RDMS)-certified emergency physician-performed identification of cholecystitis by ultrasound. J Emerg Med 2010;38 (05):645-651

15 Ratanaprasatporn L, Uyeda JW, Wortman JR, Richardson I, Sodickson AD. Multimodality imaging, including dual-energy CT, in the evaluation of gallbladder disease. Radiographics 2018;38(01):75-89

16 Yamin Z, Xuesong B, Guibin Y, Liwei L, Fei L. Risk factors of gallbladder polyps formation in East Asian population: a metaanalysis and systematic review. Asian J Surg 2020;43(01):52-59

17 Yin SN, Chi J, Liu L, Ding N, Ji YD, Yuan JM. Dual-energy CT to differentiate gallbladder polyps: cholesterol versus adenomatous. Acta Radiol 2021;62(02):147-154

18 Hundal R, Shaffer EA. Gallbladder cancer: epidemiology and outcome. Clin Epidemiol 2014;6:99-109

19 Misra S, Chaturvedi A, Misra NC, Sharma ID. Carcinoma of the gallbladder. Lancet Oncol 2003;4(03):167-176

20 Gupta P, Meghashyam K, Marodia Y, et al. Locally advanced gallbladder cancer: a review of the criteria and role of imaging. Abdom Radiol (NY) 2021;46(03):998-1007

21 Gupta P, Kumar M, Sharma V, Dutta U, Sandhu MS. Evaluation of gallbladder wall thickening: a multimodality imaging approach. Expert Rev Gastroenterol Hepatol 2020;14(06):463-473

22 Gupta P, Marodia Y, Bansal A, et al. Imaging-based algorithmic approach to gallbladder wall thickening. World J Gastroenterol 2020;26(40):6163-6181

23 Gupta P, Dutta U, Rana P, et al. Gallbladder reporting and data system (GB-RADS) for risk stratification of gallbladder wall thickening on ultrasonography: an international expert consensus. Abdom Radiol (NY) 2022;47(02):554-565

24 Wang L, Zhang Y, Chen Y, et al. The performance of a dual-energy $\mathrm{CT}$ derived radiomics model in differentiating serosal invasion for advanced gastric cancer patients after neoadjuvant chemotherapy: iodine map combined with $120-\mathrm{kV}$ equivalent mixed images. Front Oncol 2021;10:562945

25 Zhao H, Li W, Huang W, et al. Dual-energy CT-based nomogram for decoding HER2 status in patients with gastric cancer. AJR Am J Roentgenol 2021;216(06):1539-1548

26 Wang N, Ju Y, Wu J, et al. Differentiation of liver abscess from liver metastasis using dual-energy spectral CT quantitative parameters. Eur J Radiol 2019;113:204-208 